\title{
$\alpha$-Mangostin Treats Early-Stage Adjuvant-Induced Arthritis of Rat by Regulating the CAP-SIRTI Pathway in Macrophages
}

\author{
Wen-Gang Chen ${ }^{1} *$, Sa-Sa Zhang ${ }^{1-3, *}$, Shu Pan ${ }^{1-3}$, Zhong-Fang Wang', Jin-Ying Xu', Xue-He Sheng', \\ Qin Yin ${ }^{1,2}$, Yi-Jin $W u^{1,3}$
}

'Department of Pharmacy, The Second Affiliated Hospital of Wannan Medical College, Wuhu, 241000, Anhui, People's Republic of China; ${ }^{2}$ Graduate School, Wannan Medical College, Wuhu, 241000, Anhui, People's Republic of China; ${ }^{3}$ Xin'An Medicine Research Center, Wannan Medical College, Wuhu, 241000, Anhui, People's Republic of China

*These authors contributed equally to this work

Correspondence: Qin Yin; Yi-Jin Wu, Department of Pharmacy, The Second Affiliated Hospital of Wannan Medical College, Wuhu, 24I000, Anhui, People's Republic of China, Tel +86 13905530299; +86 17318531920, Email yq0553@I63.com; yijinwul224@I63.com

Background: Studies have found that $\alpha$-mangostin (MG) can relieve experimental arthritis by activating cholinergic antiinflammatory pathway (CAP). It affects the polarization of macrophages and the balance of related immune cell subpopulations, but the specific mechanism is still unclear. It has been found that silent information regulator 1 (SIRT1) is closely related to macrophage activity. The purpose of this study is to explore the mechanism of MG intervening in macrophage polarization during treatment of early adjuvant-induced (AIA) rats through the CAP-SIRT1 pathway.

Methods: We investigated the polarization of M1 macrophages and the differentiation of Th1 in AIA rats by flow cytometry. Activity of acetylcholinesterase (AChE) and the level of nicotinic adenine dinucleotide (NAD+) in serum were also detected, and immunohistochemical was used to detect the levels of $\alpha 7$ nicotinic cholinergic receptor $(\alpha 7 \mathrm{nAChR})$ and SIRT1. Then in macrophages, the molecular mechanism of MG regulating the abnormal activation of macrophages in rats with early AIA through the CAP-SIRT1 pathway was studied.

Results: MG can significantly inhibit the polarization of M1 macrophages and the differentiation of Th1 in AIA rats in the acute phase of inflammation. MG can significantly inhibit the activity of AChE and increase the level of NAD+, thereby further up-regulated the

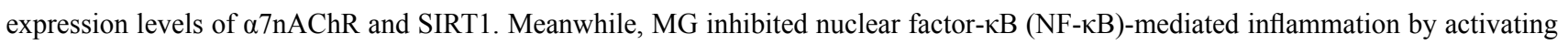
the CAP-SIRT1 pathway in macrophages.

Conclusion: In summary, the stimulation of MG induced CAP activation, which up-regulated SIRT1 signal, and thereby inhibited M1 polarization through the NF- $\mathrm{B}$ pathway, and improved the pathological immune environment of early-stage AIA rats.

Keywords: $\alpha$-mangostin, rheumatoid arthritis, choline anti-inflammatory pathway, silent information regulator 1 , macrophages

\section{Introduction}

Rheumatoid arthritis (RA) is a systemic autoimmune disease with high disability rate, which seriously affects the labor ability and quality of life of patients. ${ }^{1}$ More and more evidences show that the development of RA is the result of immune cells abnormalities. ${ }^{2}$ The phenomenon that a large number of macrophages infiltrated in the synovium of RA patients is regarded as an important sign of early RA, and their number is closely related to disease activity. ${ }^{3}$ At present, it is generally believed that 1-2 months in the initial stage of RA is the "window period" of treatment, and effective response will significantly improve the prognosis. ${ }^{4,5}$ According to this consensus, biological therapies targeting cytokines involved in macrophage-related immune responses, such as interleukin $1 \beta$ (IL-1 $\beta$ ) and tumor necrosis factor- $\alpha$ (TNF- $\alpha$ ), have been applied successfully to RA treatments. However, it is difficult to restore the overall balance of the complex 
cytokine network with one single or a few targeted drugs in combination therapy, and the high price is also an important factor restricting its application. ${ }^{6}$

In recent years, great progress has been made in understanding the association of cholinergic systems with immunity and inflammation. Cholinergic anti-inflammatory pathway (CAP) is an important functional branch of cholinergic nerve discovered in recent years. The basic molecular mechanism of CAP activation is based on the stimulation of $\alpha 7$ nicotinic cholinergic receptor $(\alpha 7 \mathrm{nAChR})$ by acetylcholine $(\mathrm{ACh})$ regulated and released by autonomic nerves. ${ }^{7,8}$ Recent studies have shown that CAP stimulation has great potential in the treatment of inflammatory diseases. Electrical stimulation of the vagus nerve or stimulation with $\alpha 7 \mathrm{nAChR}$ selective agonists can effectively alleviate the clinical symptoms of RA and corresponding animal models. ${ }^{9-11}$ Silent information regulator 1 (SIRT1), a third type of histone deacetylase dependent on nicotine adenine dinucleotide (NAD+), as an important regulatory center of energy metabolism, ${ }^{12}$ is theoretically affected by CAP. In view of the far-reaching effects of CAP on immunity and inflammation, anti-rheumatic therapy targeting CAP has a bright future, although there is currently no such drug. Identifying CAP agonists from traditional drugs or natural products is a feasible way to achieve a breakthrough in RA treatment.

Our previous research found that xanthone derivatives (XANs) can restore the cytokine composition in arthritis model animals, reduce the level of oxidative stress, and inhibit the abnormal proliferation of FLS. ${ }^{13,14}$ Among investigated compounds, $\alpha$-mangostin (MG, an isoprenyl-substituted xanthone isolated from mangosteen) exhibits particularly significant anti-rheumatic effects. ${ }^{15,16}$ We also found that MG induced the excitement of cholinergic nerves in rats by promoting the expression of $\alpha 7 \mathrm{nAChR}$, thereby reducing the severity of acute lung injury caused by LPS. ${ }^{17}$ Further studies have shown that the up-regulation of CAP of peripheral monocytes by MG can improve the systemic immune environment, restore the Th17/Treg ratio, and reduce the release of pro-inflammatory factors, exerting a therapeutic effect on arthritic rats. ${ }^{18}$ In addition, our recent research has found that MG can effectively inhibit the body's energy metabolism, which is prominently manifested as a down-regulation of nicotinamide phosphoribosyltransferase (NAMPT)/NAD. Based on the widely recognized energy-immune feedback mechanism, this phenomenon has farreaching significance for the treatment of RA and other immune-related diseases. As an NAD+-dependent enzyme, SIRT1 must also be affected by MG stimulation during this process.

Macrophages, as the main body of the innate immune system, will inevitably induce lymphocyte imbalance in their functional state, thereby promoting the development of secondary symptoms. ${ }^{19}$ Therefore, the effect of MG on the immune function of RA patients by regulating macrophages through CAP-SIRT1 is of great significance. In this study, we further verified the stimulating effect of MG on the CAP-SIRT1 pathway in macrophages, and preliminarily clarified its correlation with the therapeutic effect of AIA in rats.

\section{Materials and Methods}

\section{Materials and Reagents}

MG (purity $>98 \%$ ) was purchased from SanHerb Bioscience (Chengdu, Sichuan, China). Carboxyl methyl cellulose sodium (CMC-Na) was obtained from Aladdin (Shanghai, China). Lipopolysaccharide (LPS, from the Gram-negative bacterium E. coli 055:B5) and methyllycaconitine (MLA, $\alpha 7 \mathrm{nAChR}$ specific inhibitor) were purchased from Sigma-Aldrich (St. Louis, MO, USA). Roswell Park Memorial Institute (RPMI) 1640 medium and Dulbecco's modified Eagle's medium (DMEM) were bought from Hyclone (Logan City, UT, USA). Fetal bovine serum (FBS) was purchased from Gibco (CA, USA). Lymphocyte separation solution was bought from Solarbio (Beijing, China). Anti-rat CD4-PE, IFN- $\gamma$-FITC, CD86-FITC and CD11b-PE antibodies were purchased from BioLegend (San Diego, CA, USA). Anti-rat p65, ac-p65, p-p65, $\alpha 7 n$ AChR and SIRT1 antibodies were the products of Affinity Biosciences (Changzhou, Jiangsu, China). Biotin-conjugated secondary antibodies were supplied by Beyotime Biotech (Nantong, Jiangsu, China). Acetylcholinesterase (AChE) assay kit and Coenzyme I NAD (H) assay kit were supplied by JianCheng Bioengineering Institute (Nanjing, Jiangsu, China).

\section{Cells and Animals}

RAW264.7 cells were purchased from American Type Culture Collection (ATCC number: TIB-71, USA). Male Sprague-

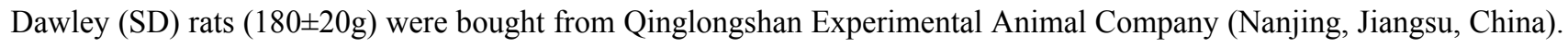


The animals were acclimated for 7 days prior to experimental procedures. The animal experimental protocols were approved by the Ethical Committee of The Second Affiliated Hospital of Wannan Medical College (no. wyefy 2018-004), strictly in accordance with the Guidelines for the Care and Use of Laboratory Animals (United States National Research Council, 2011).

\section{Induction of Adjuvant-Induced (AIA) in Rats and Treatments}

Bovine type II collagen (Chondrex, Redmond, WA, USA) for immunization was dissolved in $0.1 \mathrm{M}$ acetic acid, stirred at $4^{\circ} \mathrm{C}$ and fully dissolved to prepare a solution with a concentration of $2 \mathrm{~g} / \mathrm{L}$. The solution was then mixed into complete Freund's adjuvant (CFA) dropwise, and fully emulsified in an ice-water bath to obtain a milky homogeneous emulsion. The emulsion was used to implement multiple intradermal injections $(0.2 \mathrm{~mL})$ on the back and tail roots of SD rats, which was recorded as the $0 \mathrm{~d}$, and the $7 \mathrm{~d}$ was used to enhance immunity. After the first injection, 18 immunized rats were randomly divided into AIA group, MG early treatment group (0-20d) and MG late treatment group (21-40d). Another 6 healthy rats were taken as normal control group. MG was dissolved in $0.5 \% \mathrm{CMC}-\mathrm{Na}$ with the help of ethanol and Tween 40, and was administered to rats by gavage at a dose of $40 \mathrm{mg} / \mathrm{kg}$ per day. The normal and AIA groups were treated simultaneously with the same amount of $0.5 \% \mathrm{CMC}-\mathrm{Na}{ }^{15}$

\section{Histopathological and Immunohistochemical Evaluation}

The rats were sacrificed on day 45 then the hind ankle joints and spleen were fixed in $4 \%$ formaldehyde solution. After 14 days of decalcification treatment, the joint tissues were transparentized. Then the specimens were embedded in paraffin, sectioned and stained with hematoxylin-eosin (HE). The pathological changes of the joints and spleen were observed under a light microscope and scored with the same criteria defined previously. ${ }^{20-22}$

We dewaxed the paraffin sections and immersed them in a methanol solution of $\mathrm{H}_{2} \mathrm{O}_{2}$ to inactivate endogenous peroxidase. The slices were heated by microwave and repaired by citric acid, then incubated with corresponding primary and secondary antibodies in sequence. The signal was then visualized by diaminobenzidine (DAB) chromogenic solution and hematoxylin (Zsbio, Beijing, China). Stained sections were finally dehydrated, sealed, and observed under a light microscope. ${ }^{22}$

\section{Cell Harvest and Culture}

Macrophages were obtained from the abdominal cavity of each group of rats. $10 \mathrm{~mL}$ of serum-free DMEM were injected into the abdominal cavity of rats, and the abdomen was massaged for 2-3 minutes and then left for 5 minutes to enrich the macrophages. Then, we aspirated the cell suspension, centrifuged at $2500 \mathrm{rpm}$ for 10 minutes, and added $10 \%$ high sugar DMEM to resuspend it in a six-well plate. After the cells had been cultured for $2 \mathrm{~h}$, we discarded the supernatant and washed three times with sterile PBS. The attached macrophages are purified macrophages. ${ }^{23}$

The peripheral blood mononuclear cells (PBMCs) of each group of rats were prepared according to the following steps. Fresh blood was diluted with pre-cooled RPMI1640, then added to a $15 \mathrm{~mL}$ centrifuge tube. We mixed the diluted blood with an equal volume of peripheral blood lymphocyte separation solution. The diluted blood was slowly spread on the upper layer of the separation solution, and the PBMCs were separated from the whole blood by gradient centrifugation. ${ }^{18}$

In addition, RAW264.7 cells were cultured in a six-well plate with a density of $2 \times 10^{5} /$ well, and DMEM medium containing $20 \%$ FBS was adopted in the culture system.

After different stimuli, cells can be divided into control, LPS ( $2 \mathrm{nM})$, MLA ( $5 \mathrm{nM}), \mathrm{MG}(5 \mu \mathrm{g} / \mathrm{mL})$ and MLA+MG. After the cells were cultured for $24 \mathrm{~h}$, the supernatant was discarded and the cells were washed twice with pre-cooled PBS. The cells were centrifuged at $12,000 \mathrm{rpm}$ at $4^{\circ} \mathrm{C}$ for 10 minutes, and the supernatant was taken as the protein sample for the next experiment.

\section{Evaluation of Activity of AChE and Level of NAD+ in Serum Under MG Treatments}

Before the test, serum was obtained from the whole blood of each group of rats by centrifugation. The activity of AChE in serum was directly detected by AChE assay kit. The absorbance of the tested sample was read at $412 \mathrm{~nm}$, and the read was used for the calculation of AChE activity according to the quantitative formula given in the manual. 
Extraction of NAD + in serum: serum was mixed proportionally with the acidic extract in the kit, boiled for 5 minutes, cooled in an ice bath, and centrifuged at $10000 \mathrm{~g}$ for 10 minutes at $4^{\circ} \mathrm{C}$. The supernatant was transferred to another new centrifuge tube, and an equal volume of alkaline extract was added and mixed. The supernatant was centrifuged at $10000 \mathrm{~g}$ at $4^{\circ} \mathrm{C}$ for $10 \mathrm{~min}$, and the supernatant was taken for detection. The absorbance of the tested sample was read at $570 \mathrm{~nm}$, and the read was used for the calculation of NAD+ activity according to the quantitative formula given in the manual.

\section{Western Blot Assay}

Qualified protein samples (10 $\mu \mathrm{g} /$ well, based on the BCA test) were subjected to sodium dodecyl sulfate-polyacrylamide gel electrophoresis (SDS-PAGE). Separated proteins were transferred to a polyvinylidene fluoride (PVDF) membrane, which were then incubated with primary antibodies (p65 anti-rabbit, 1:1000; p-p65 anti-rabbit, 1:1000; ac-p65 antirabbit, 1:1000; SIRT1 anti-rabbit, 1:1000; $\alpha 7 \mathrm{nAChR}$ anti-rabbit, 1:1000; $\beta$-actin anti-rat, $1: 3000$ ) overnight at $4^{\circ} \mathrm{C}$, followed by blocking with 5\% skim milk for $2 \mathrm{~h}$ at room temperature. Followed by incubation with a specific antigenlabeled secondary antibody. Finally, signals were developed with an enhanced chemiluminescence (ECL) substrate kit. The results were photographed and analyzed using ImageJ software.

\section{Flow Cytometry}

The macrophages of rats in each group were obtained according to the above method. CD11b antibodies were added to cells and protected from light for 30 minutes. After cells were fixed and membrane permeabilized, different fluorescently labeled CD86 antibodies were added and incubated for 1 hour at room temperature. Treated cells were washed with PBS and centrifuged at $2500 \mathrm{rpm} / 10 \mathrm{~min}$. The supernatant was removed and resuspended in PBS. Similarly, PBMC was obtained according to the above method. Surface antibodies CD4 were added, and the membrane was fixed and permeabilized. Different fluorescein-labeled IFN- $\gamma$ antibodies were added and incubated for $30 \mathrm{~min}$ in the dark, washed and resuspended. The stained cells were subsequently fed to a flow cytometer (FC500, Beckman) for quantitative analysis. Obtained data were analyzed by FlowJo (version 10). $\mathrm{CD}_{11} \mathrm{~b}^{+} \mathrm{CD} 86^{+}$and $\mathrm{CD} 4^{+} \mathrm{IFN}-\gamma^{+}$cells were identified as M1 and Th1 cells, respectively.

\section{Data Analysis}

The data were typically expressed as mean \pm standard deviation (SD) (Supplementary File 1). Data distribution was evaluated by Shapiro-Wilk test and P-P plot (Supplementary File 2), then statistical differences among different groups were evaluated using one-way analysis of variance followed by the Tukey post hoc tests using the SPSS 16.0 software. The Kruskal-Wallis $H$-test followed by the Dunn's test was used for skewed data. The skewed data were presented as median (min - max) (Supplementary File 1). Differences were considered statistically significant when $P<0.05$. All the statistical analyses were performed with GraphPad Prism 8.0 (Cary, NC, USA).

\section{Results}

\section{The Effect of MG Periodic Treatment on the Severity of Inflammation in AIA Rats}

As shown in Figure 1A, hind paws of AIA rats suffered from severe inflammation, which was not obvious in MG (early) and MG (late) treated rats. Comparatively, arthritic condition of MG (late)-treated rats was worse than MG (early) counterparts, as joints deformation and bulbous inflammation can be still noticed in their paws. This provided basic evidence for the anti-rheumatic properties of MG in vivo.

In the histological examination, compared with normal rats, AIA rats showed excessive proliferation of joint synovial cells, massive infiltration of inflammatory cells, pannus formation and articular cartilage erosion. MG ameliorated all the pathological changes effectively (Figure 1B and D). In addition, there were abnormal changes in the spleen of AIA rats, including the blurring of the white pulp and the red pulp, and the hyperplasia of the white pulp. At the same time, the increased density of lymphocytes around the germinal center artery and the obvious hyperplasia of the red pulp can be seen. After treatment with MG, the number of germinal centers was significantly reduced and the boundary between 

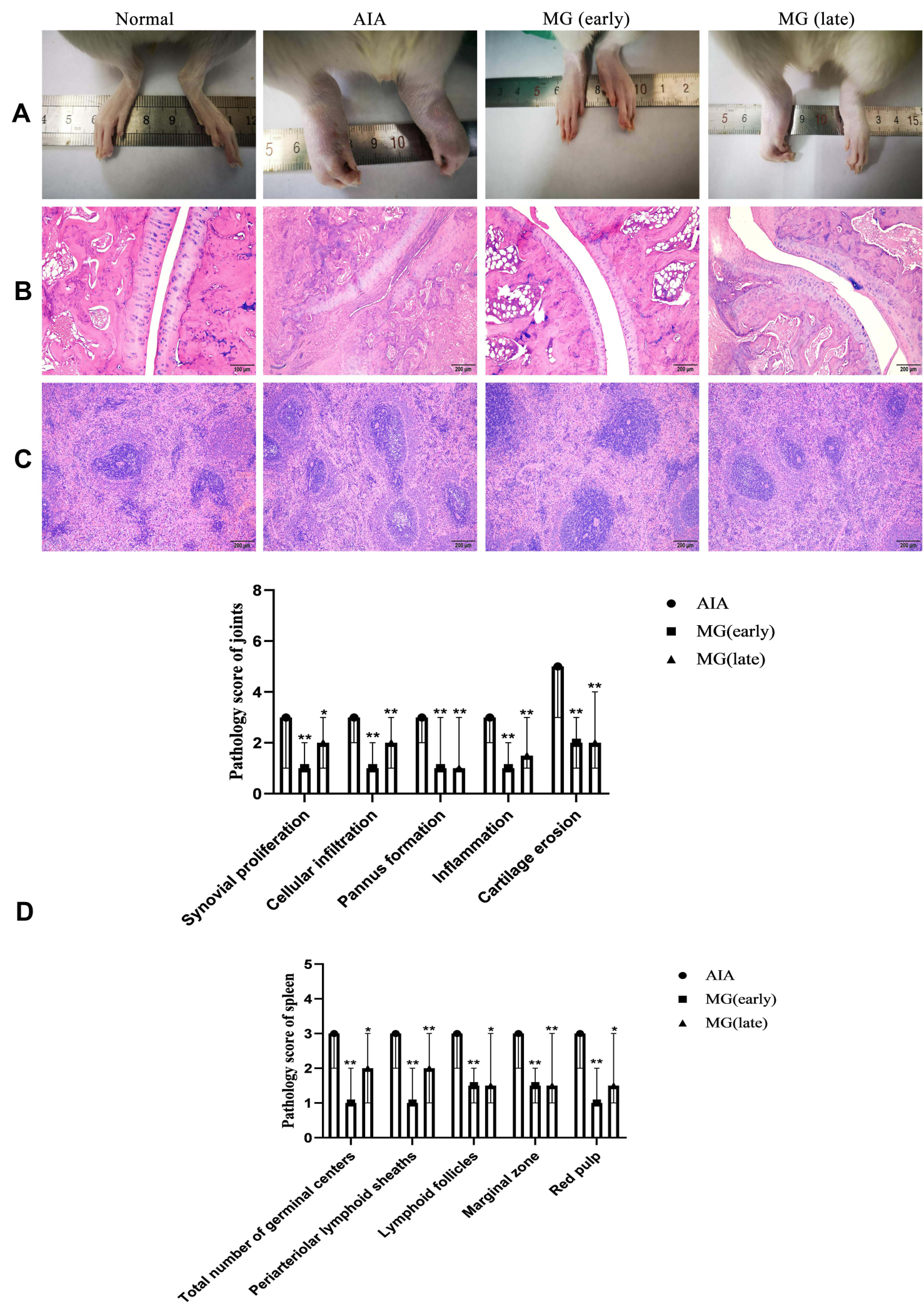

Figure I Therapeutic effects of MG on AIA rats. (A) Morphological observation of hind paw (B) histological examination of joint; (C) histological examination of spleen; (D) histopathological scores of joint and spleen. $* P<0.05$ and $* * P<0.01$ compared with AIA rats. 
white and red pulp was significantly improved (Figure $1 \mathrm{C}$ and $\mathrm{D}$ ). The above facts show that $\mathrm{MG}$ treatment can reduce the pathological score, and thereby improved the pathological manifestations. And the therapeutic effect of MG (early) is slightly better than MG (late).

\section{MG Inhibited Polarization of MI Macrophages in AIA Rats}

The phenomenon that a large number of macrophages infiltrated in the synovium is regarded as important signs of the early stage of RA, and their number is closely related to the disease activity. Macrophages as the main composition of the innate immune system, their functional state disorder will inevitably induce lymphocyte imbalance, and thereby promote the development of secondary symptoms. ${ }^{5}$ Because the increase of M1 macrophages and Th1 cells plays an important role in the occurrence and development of RA. ${ }^{24,25}$ In this study, we used flow cytometry to investigate the effects of MG on macrophage polarization and peripheral $\mathrm{T}$ lymphocyte differentiation during the acute and secondary inflammation in AIA rats. As anticipated, M1 macrophages were increased significantly in the AIA rats. Both the MG (early) and the MG (late) significantly reduced its distribution, from $22.20 \%$ to $9.51 \%$ and $11.50 \%$, respectively, and MG (early) has a stronger effect on inhibiting M1 polarization (Figure 2A and C). Accordingly, compared with normal rats, Th1 subset in AIA rats was increased significantly. Both the MG (early) and the MG (late) significantly reduced its distribution, from $29.20 \%$ to $8.44 \%$ and $11.80 \%$, respectively, and MG (early) has a stronger effect on inhibiting Th1 differentiation (Figure $2 \mathrm{~B}$ and $\mathrm{D}$ ). The above clues preliminarily suggest that $\mathrm{MG}$ has a significant inhibitory effect on M1 polarization in the acute phase of inflammation in AIA rats. This property is meaningful for subsequent inflammation alleviation and immune homeostasis restoration.

\section{MG Activated the CAP-SIRTI Pathway in Macrophages}

Our previous study found that MG can induce the activation of CAP in vivo and in vitro, and the selective CAP can partially reverse the anti-inflammatory effect of MG (data not provided). At the same time, we also found that MG can effectively inhibit the body's energy metabolism, which is prominently manifested by down-regulation of NAMPT/ NAD. ${ }^{7}$ Vagus nerve activation and up-regulation of CAP are orchestrated in energy metabolism. SIRT1 is regarded as the core node of the cross-talk between energy metabolism and immune inflammation, which must be affected by the state of CAP in theory. Therefore, we simultaneously analyzed the status of the CAP-SIRT1 pathway to further clarify the molecular mechanism of MG intervention in macrophage polarization during treatment of the acute inflammation of AIA.

The results show that $\alpha 7 \mathrm{nAChR}$ was mainly expressed on lymphocytes located in the medullary part of the spleen (Figure 3A). After MG treatment, this decline in the acute inflammation phase was effectively restored, which was especially notable during acute inflammation (Figure 3C). This result demonstrates the positive effects of MG on the CAP in the immune system in vivo. Similarly, SIRT1 is also mainly distributed in the medulla of the spleen (Figure 3B) and significantly reduced in AIA rats. MG treatment reversed this abnormality (Figure 3D). The decrease in $\alpha 7 \mathrm{nAChR}$ and SIRT1 in AIA rats was accompanied by a significant increase in AChE activity and a decrease in NAD+ levels. It seems that MG has a regulatory effect on CAP-SIRT1, because the above abnormal changes have recovered to varying degrees after MG treatment (Figure 3E and F).

Based on the above results, we speculated that MG may stimulate $\alpha 7 \mathrm{nAChR}$ to activate $\mathrm{CAP}$, and then further upregulate SIRT1 signal, which will improve the immune environment in the acute inflammation phase of AIA. Therefore, we isolated macrophages from each group of rats for further verification. The result echoes the result of spleen immunohistochemistry. Compared with normal, the expression of $\alpha 7 \mathrm{nAChR}$ and SIRT1 in macrophages of AIA rats was significantly decreased (Figure $4 \mathrm{~A}$ ). $\mathrm{MG}$ can reverse the low expression of $\alpha 7 \mathrm{nAChR}$ and SIRT1 in AIA rats (Figure 4B and C). Since the overactivation of nuclear factor- $\mathrm{KB}(\mathrm{NF}-\mathrm{\kappa B})$ is closely related to the polarization of macrophages, its changes are essential to alleviate the joint inflammation of AIA/RA. As expected, MG treatment inhibited the phosphorylation of p65 in macrophages of AIA rats (Figure 4A and D). In addition, SIRT1 is a deacetylase deacetylating a variety of substrates. We also investigated the deacetylation effect of SIRT1 on p65. Compared with AIA rats, the high expression of SIRT1 induced by MG is accompanied by enhanced deacetylation of p65 (Figure 4A and E). 

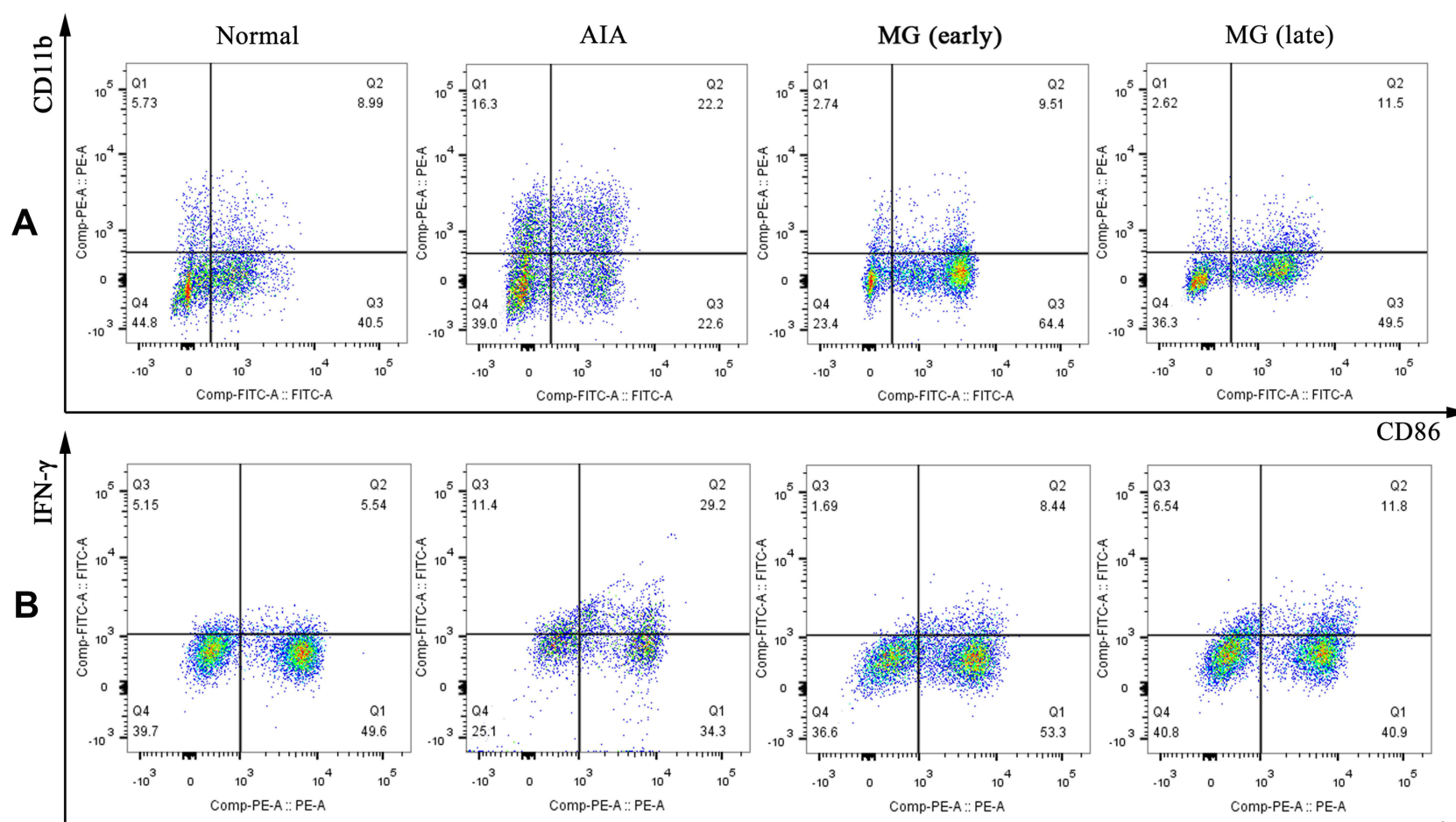

CD86

C

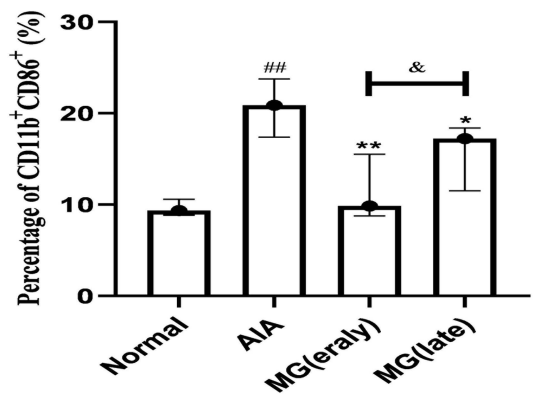

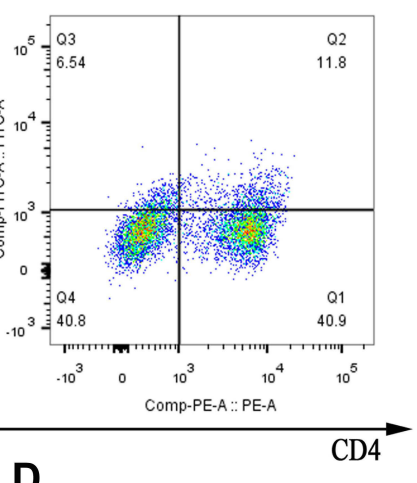

D

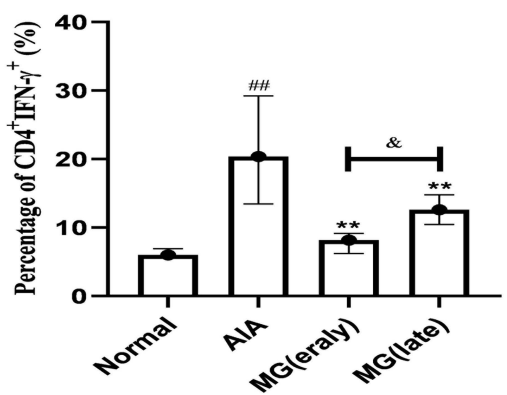

Figure 2 Effects of MG on the polarization of MI macrophages and ThI distribution of PBMCs in AIA rats were explored by flow cytometry. (A) Representative raw data indicating the polarization of MI macrophages; (B) representative raw data indicating the ThI distribution; (C and $\mathbf{D})$ quantification results of flow cytometry experiments. ${ }^{\#} P<0.0$ l compared with normal, ${ }^{*} P<0.05$, ${ }^{* *} P<0.01$ compared with AIA rats, ${ }^{\&} P<0.05$ compared with MG (early).

Based on the above clues, we believe that MG may act on the abnormal polarization of M1 macrophages in the acute inflammation phase of AIA through the CAP-SIRT1 pathway, and affect the development and prognosis of the disease.

\section{MG Relieved LPS-Induced Inflammation by Regulating $\alpha 7 n A C h R$ in RAW264.7 Cells to Stimulate SIRTI}

Based on the above results, in order to further explore the regulatory effect of CAP on SIRT1 under inflammatory conditions, we used MLA to selectively inhibit $\alpha 7 \mathrm{nAChR}$ and then antagonize CAP. The results show that compared to control, LPS can significantly inhibit the expression of $\alpha 7 \mathrm{nAChR}$ and SIRT1 (Figure 5A). Similarly, MLA can significantly inhibit the expression of $\alpha 7 \mathrm{nAChR}$ (Figure 5A and B), and the expression of SIRT1 is also downregulated accordingly (Figure 5A and C). Compared with LPS, MG can up-regulate the expression levels of $\alpha 7 \mathrm{nAChR}$ and SIRT1. Importantly, MG can also reverse the down-regulation of $\alpha 7 \mathrm{nAChR}$ and SIRT1 levels caused by MLA (Figure 5). Therefore, we can preliminarily conclude that MG stimulates the expression of SIRT1 at least partially through $\alpha 7 \mathrm{nAChR}$ activation. 


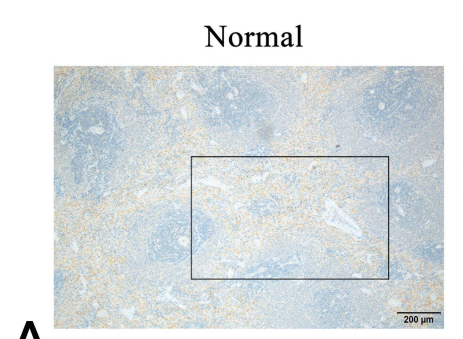

A
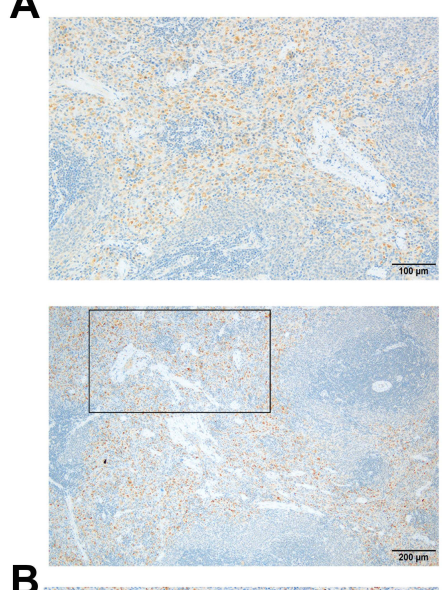

B

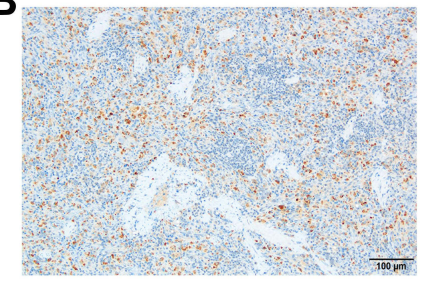

C

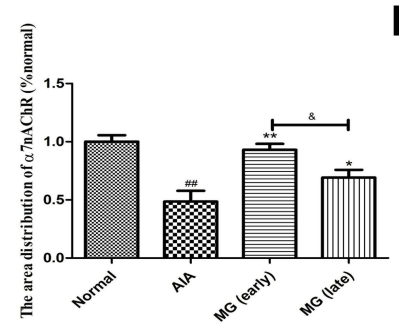

AIA
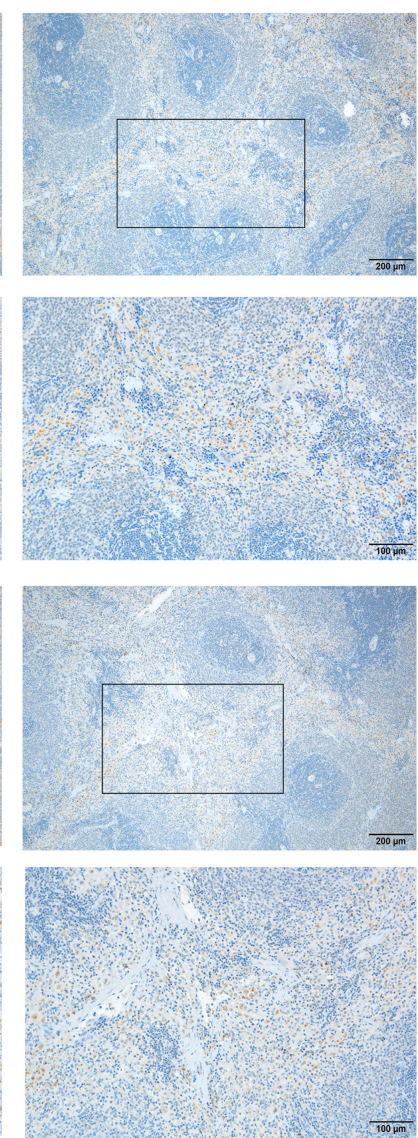

$D_{-1}$

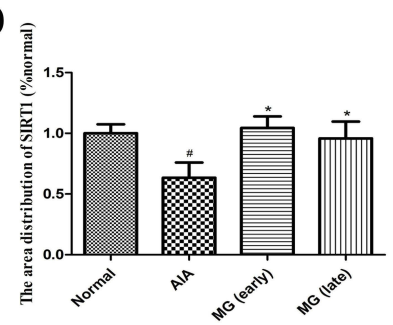

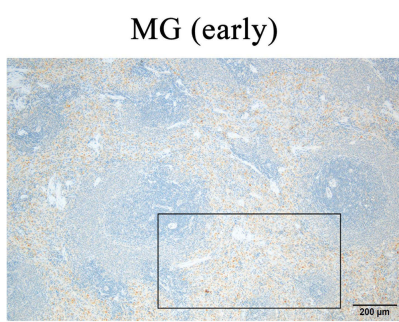
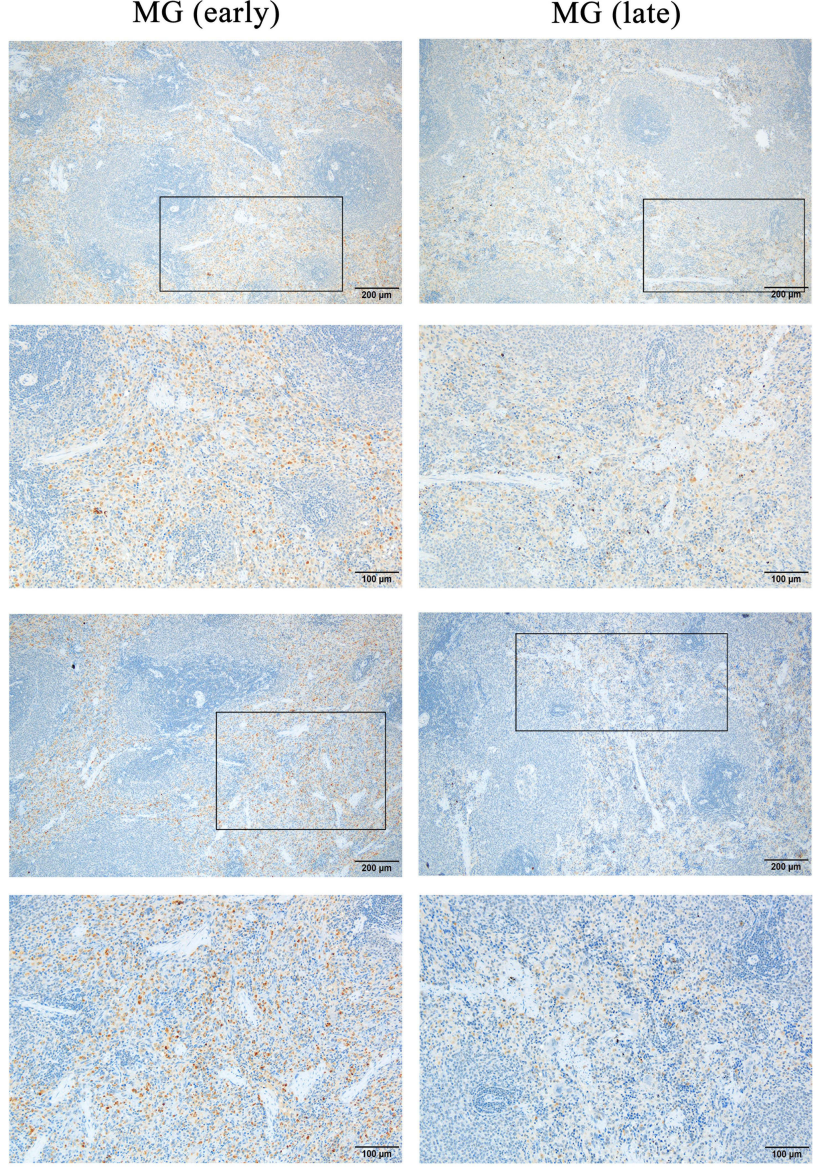

$\mathbf{E}_{\text {言 }}$
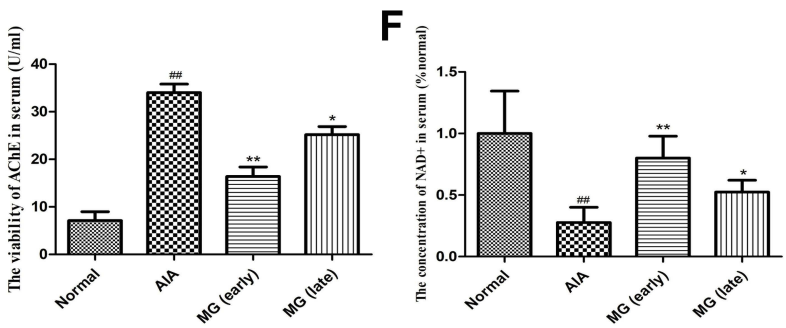

Figure 3 Possible effects of MG on CAP-SIRTI in AIA rats. (A) Local expression of $\alpha 7 n A C h R$ in the spleen; (B) local expression of SIRTI in the spleen; (C and D) quantification results of the immunohistochemical experiment analyzed by ipwin software; (E) inhibitory effect of $M G$ on enzymatic activity of $A C h E$ in serum; (F) MG increases the concentration of NAD+ in the serum. ${ }^{\#} P<0.05,{ }^{\#} P<0.01$ compared with normal rats, ${ }^{*} P<0.05$, ${ }^{* * P} P<0.01$ compared with AIA rats, ${ }^{\circledR} P<0.05$ compared with $M G$ (early).

\section{Discussion}

MG is a natural polyphenol, mainly distributed in the peel of mangosteen. Due to its remarkable biological activity and high abundance, the exploration of its medicinal potential is not only clinically significant, but also economically important. ${ }^{26}$ Previous studies have shown that $\mathrm{MG}$ and its extract have remarkable effects on experimental arthritis model rats. ${ }^{15,16}$ In addition to the improvement of arthritis indicators, the above treatments have a profound impact on the immune microenvironment. For example, the expression of Toll-like receptors 4 (TLR4) and p-p65 in joints is greatly reduced, and as well as the distribution of Th1 cells. TLR4/NF- $\mathrm{kB}$ activation is a key event in the polarization of M1 macrophages. The above clues preliminarily suggest that MG has an inhibitory effect on M1 polarization under active RA conditions. Although it is generally believed that the pathological changes of RA are mainly controlled by lymphocytes, especially CD4+ T cells. But the role of macrophages in RA cannot be ignored. As an indispensable part of innate immunity, these cells first sense pathogens and transmit signals to the adaptive immune system. In addition, 
A

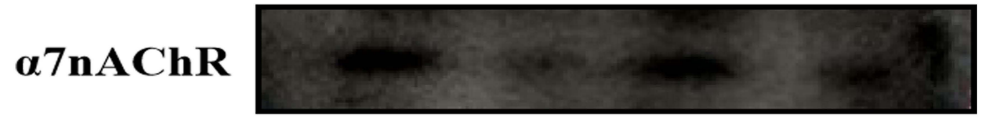

Sirt1

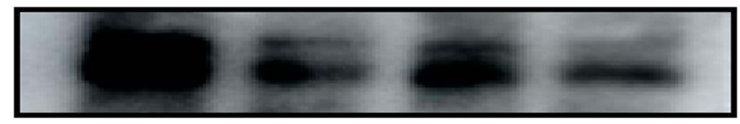

\section{ac-p65}

p-p65

p65
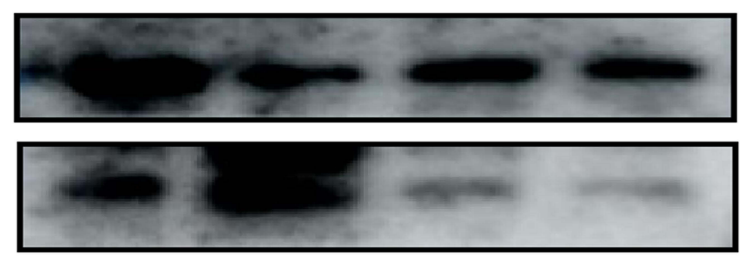

Actin
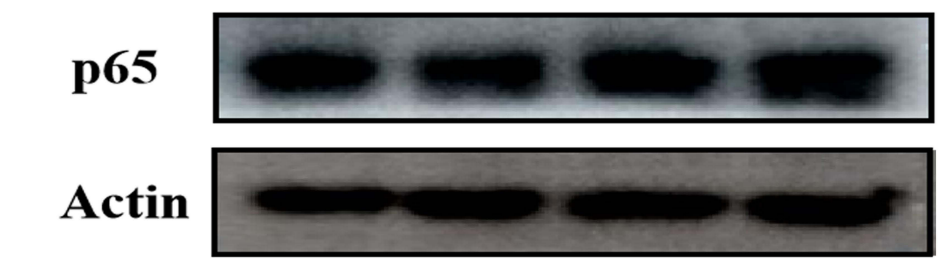

\section{Groups}

D

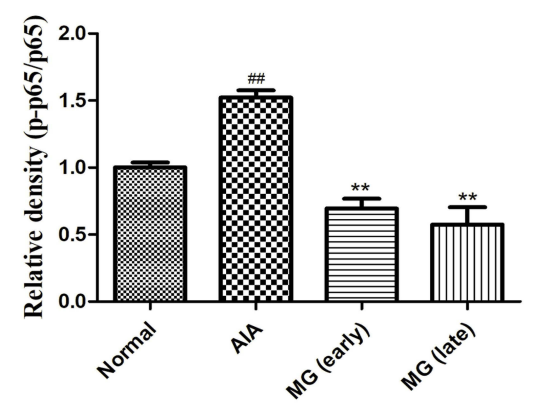

B

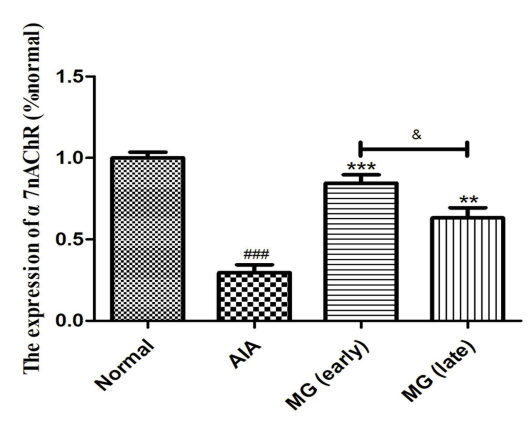

C

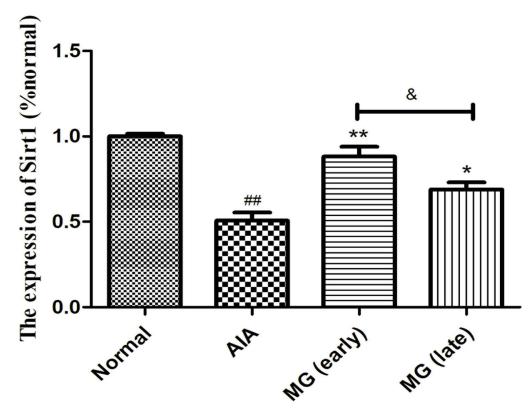

Figure 4 Effect of MG activated CAP-SIRTI pathway on NF-kB-mediated inflammatory response in rat macrophages. (A) Expression of $\alpha 7 n A C h R$, SIRTI, ac-p65, p-p65 and

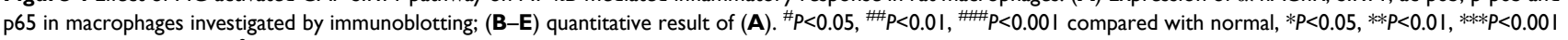
compared with AIA rats, ${ }^{\&} P<0.05$ compared with MG (early).

M1 polarization is closely related to the manifestations of arthritis, including persistent inflammation and progressive tissue injury of joints. The imbalance of macrophage polarization may be the main reason for the deterioration of immune environment in RA patients. M1 macrophages are the main promoting factor of Th1 differentiation. From this perspective, the effects of MG on M1 polarization and Th1 differentiation in AIA rats revealed by this study are very meaningful and enlightening.

Our early research shows that this phenomenon is closely related to CAP activation. Electrical stimulation of the vagus nerve or stimulation with $\alpha 7 \mathrm{nAChR}$ selective agonists can effectively alleviate the clinical symptoms of RA and corresponding animal models. CAP activation is accompanied by a significant increase in M2 polarization and a decrease in M1 polarization. ${ }^{9}$ Macrophages are the main immune cells highly expressing $\alpha 7 n A C h R$ in the periphery. Further studies have shown that the up-regulation of CAP of peripheral monocytes by MG can improve the systemic immune environment, restore the Th17/Treg ratio, and reduce the release of pro-inflammatory factors, demonstrating a therapeutic effect on CIA rats. ${ }^{18}$ Therefore, the activation of CAP by MG in RA is beneficial to suppress the abnormal polarization of M1 macrophages. In order to verify the above speculation, it is imperative to clarify the effect of MG on CAP in the immune system. On the one hand, MG treatment greatly inhibits the catalytic activity of AChE, and the impaired hydrolysis will eventually lead to slowed clearance of $\mathrm{ACh}$, due to the prolonged CAP activation, it will elicit antiinflammatory effects. On the other hand, we found that MG can effectively inhibit the body's energy metabolism, which is prominently manifested by down-regulation of NAMPT/NAD. Based on the widely recognized energy-immune 
A

a7nAChR

Sirt1

Actin

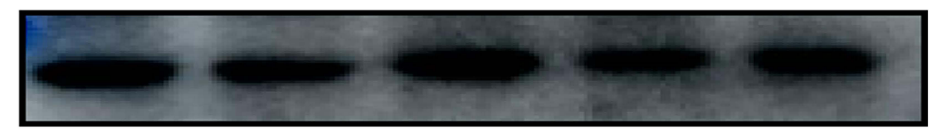

LPS

- $\quad+$

$+$

$+$

$+$

MLA

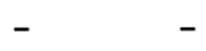

$-\quad+$

$\begin{array}{ll}- & +\end{array}$

MG

B

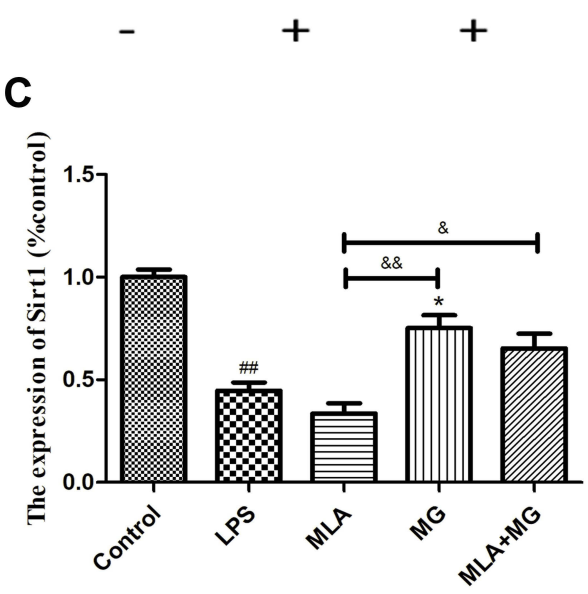

Figure 5 MG relieves LPS-induced inflammation by regulating $\alpha 7 n A C h R$ in RAW264.7 cells to stimulate SIRTI. (A) Expression of $\alpha 7 n A C h R$, SIRTI in RAW264.7 cells investigated by immunoblotting; (B and $\mathbf{C})$ quantitative result of $(\mathbf{A}) .{ }^{\#} P<0.01$ compared with control, ${ }^{*} P<0.05,{ }^{* * P}<0.01$ compared with LPS, ${ }^{\circledR} P<0.05$, ${ }^{\& \&} P<0.01$ compared with MLA.

feedback mechanism, this phenomenon has far-reaching significance for the treatment of RA and other immune-related diseases. As an NAD+-dependent enzyme, the function of SIRT1 must be affected by MG stimulation during this process. It is puzzling that previous reports and our research have found that SIRT1 is highly expressed under RA/CIA conditions, and MG negatively regulates SIRT1 in this state. Considering the spontaneous recovery of M2 macrophages development is common in RA patients at remission stages, we believe that the increased expression of SIRT1 in the late stage of RA/CIA is a part of self-balancing immune mechanism. MG may accelerate the pre-disease SIRT1-dependent M2 polarization process, thereby weakening the demand for spontaneous SIRT1 upregulation.

As mentioned above, CAP activation inhibits M1 polarization. It is worth noting that this process is accompanied by a switch from glycolysis to aerobic oxidation. Like its upstream NAMPT, SIRT1 also plays a key role in metabolic reprogramming. More importantly, the results in this study indicate that NF- $\mathrm{KB}$ activation, a key step in M1 polarization, is also controlled by SIRT1. Therefore, it is reasonable to believe that SIRT1 is an important downstream target of $\alpha 7 \mathrm{nAChR}$ and an important way for CAP to interfere with innate immunity. These findings suggest that the restoration of immune homeostasis under MG treatments could be the foundation for the overall therapeutic effects on experimental arthritis.

\section{Conclusion}

This study revealed that the intervention of MG on CAP is the basis of SIRT1 up-regulation, and consequently downregulated NF- $\mathrm{KB}$ pathway in macrophages and M1 polarization. On this basis, it was preliminarily elucidated the mechanism of MG intervention in macrophage polarization treatment of early AIA rats to improve the prognosis of the disease. In summary, we have proved that MG stimulates the CAP-SIRT1 pathway and improves the peripheral 
immune environment, thereby inhibiting the polarization of M1 through the NF- $\mathrm{B}$ pathway and improving the pathological immune environment of early AIA rats.

\section{Funding}

This work was supported by the Scientific Research Fund for Key Projects of Wannan Medical College (grant numbers WK2020ZF21), Research project of traditional Chinese Medicine Inheritance and innovation of Anhui Province (grant numbers 2020zcyb02), Scientific Research Project of Anhui Provincial Health Commission (grant numbers AHWJ2021b038) and Anhui Provincial Natural Science Foundation (grant numbers 2108085QH386).

\section{Disclosure}

The authors declare no conflicts of interest in this work.

\section{References}

1. Guidelli GM, Barskova T, Brizi MG, et al. One year in review: novelties in the treatment of rheumatoid arthritis. Clin Exp Rheumatol. 2015;33 (1):102-108.

2. Kung CC, Dai SP, Chiang H, Huang HS, Sun WH. Temporal expression patterns of distinct cytokines and M1/M2 macrophage polarization regulate rheumatoid arthritis progression. Mol Biol Rep. 2020;47(5):3423-3437. doi:10.1007/s11033-020-05422-6

3. Li J, Hsu HC, Mountz JD. Managing macrophages in rheumatoid arthritis by reform or removal. Curr Rheumatol Rep. 2012;14(5):445-454. doi:10.1007/s11926-012-0272-4

4. Scherer HU, Häupl T, Burmester GR. The etiology of rheumatoid arthritis. J Autoimmun. 2020;110:102400. doi:10.1016/j.jaut.2019.102400

5. Davignon JL, Hayder M, Baron M, et al. Targeting monocytes/macrophages in the treatment of rheumatoid arthritis. Rheumatology (Oxford). 2013;52(4):590-598. doi:10.1093/rheumatology/kes304

6. Venkatesha SH, Dudics S, Acharya B, Moudgil KD. Cytokine-modulating strategies and newer cytokine targets for arthritis therapy. Int J Mol Sci. 2014;16(1):887-906. doi:10.3390/ijms16010887

7. Tao M, Jiang J, Wang L, et al. $\alpha$-Mangostin alleviated lipopolysaccharide induced acute lung injury in rats by suppressing NAMPT/NAD controlled inflammatory reactions. Evid Based Complement Alternat Med. 2018;2018:5470187. doi:10.1155/2018/5470187

8. Wang H, Yu M, Ochani M, et al. Nicotinic acetylcholine receptor alpha7 subunit is an essential regulator of inflammation. Nature. 2003;421 (6921):384-388. doi:10.1038/nature01339

9. Yang YH, Li DL, Bi XY, et al. Acetylcholine inhibits LPS-induced MMP-9 production and cell migration via the $\alpha 7$ nAChR-JAK2/STAT3 pathway in RAW264.7 cells. Cell Physiol Biochem. 2015;36(5):2025-2038. doi:10.1159/000430170

10. Zhang P, Qin L, Zhang G. The potential application of nicotinic acetylcholine receptor agonists for the treatment of rheumatoid arthritis. Inflamm Res. 2010;59(6):415-417. doi:10.1007/s00011-010-0160-1

11. Peng C, Shi QP, Liu JY, et al. Alpha7 nAChR expression is correlated with arthritis development and inhibited by sinomenine in adjuvant-induced arthritic rats. Evid Based Complement Alternat Med. 2019;2019:3759304. doi:10.1155/2019/3759304

12. Kong S, McBurney MW, Fang D. Sirtuin 1 in immune regulation and autoimmunity. Immunol Cell Biol. 2012;90(1):6-13. doi:10.1038/ icb.2011.102

13. Zuo J, Xia Y, Li X, Chen JW. Xanthones from Securidaca inappendiculata exert significant therapeutic efficacy on adjuvant-induced arthritis in mice. Inflammation. 2014;37(3):908-916. doi:10.1007/s10753-014-9810-8

14. Zuo J, Xia Y, Mao KJ, Li X, Chen JW. Xanthone-rich dichloromethane fraction of Securidaca inappendiculata, the possible antirheumatic material base with anti-inflammatory, analgesic, and immunodepressive effects. Pharm Biol. 2014;52(11):1367-1373. doi:10.3109/ 13880209.2014.892143

15. Yang K, Yin Q, Mao Q, et al. Metabolomics analysis reveals therapeutic effects of $\alpha$-mangostin on collagen-induced arthritis in rats by downregulating nicotinamide phosphoribosyltransferase. Inflammation. 2019;42(2):741-753. doi:10.1007/s10753-018-0932-2

16. Zuo J, Yin Q, Wang YW, et al. Inhibition of NF- $\mathrm{KB}$ pathway in fibroblast-like synoviocytes by $\alpha$-mangostin implicated in protective effects on joints in rats suffering from adjuvant-induced arthritis. Int Immunopharmacol. 2018;56:78-89. doi:10.1016/j.intimp.2018.01.016

17. Yang Z, Yin Q, Olatunji OJ, et al. Activation of cholinergic anti-inflammatory pathway involved in therapeutic actions of $\alpha$-mangostin on lipopolysaccharide-induced acute lung injury in rats. Int J Immunopathol Pharmacol. 2020;34:2058738420954941. doi:10.1177/ 2058738420954941

18. Yin Q, Wu YJ, Pan S, et al. Activation of cholinergic anti-inflammatory pathway in peripheral immune cells involved in therapeutic actions of $\alpha$ mangostin on collagen-induced arthritis in rats. Drug Des Devel Ther. 2020;14:1983-1993. doi:10.2147/DDDT.S249865

19. Brennan F, Foey A. Cytokine regulation in RA synovial tissue: role of T cell/macrophage contact-dependent interactions. Arthritis Res. 2002;4 Suppl 3(Supp13):S177-S182. doi:10.1186/ar556

20. Germolec DR, Nyska A, Kashon M, et al. Extended histopathology in immunotoxicity testing: interlaboratory validation studies. Toxicol Sci. 2004;78(1):107-115. doi:10.1093/toxsci/kfh049

21. Shu JL, Zhang XZ, Han L, et al. Paeoniflorin-6'-O-benzene sulfonate alleviates collagen-induced arthritis in mice by downregulating BAFFTRAF2-NF-KB signaling: comparison with biological agents. Acta Pharmacol Sin. 2019;40(6):801-813. doi:10.1038/s41401-018-0169-5

22. Tang H, Wu YJ, Xiao F, et al. Regulation of CP-25 on P-glycoprotein in synoviocytes of rats with adjuvant arthritis. Biomed Pharmacother. 2019;119:109432. doi:10.1016/j.biopha.2019.109432

23. Wu MH, Shoji Y, Wu MC, et al. Suppression of matrix metalloproteinase-9 by prostaglandin E(2) in peritoneal macrophage is associated with severity of endometriosis. Am J Pathol. 2005;167(4):1061-1069. doi:10.1016/S0002-9440(10)61195-9 
24. Tardito S, Martinelli G, Soldano S, et al. Macrophage M1/M2 polarization and rheumatoid arthritis: a systematic review. Autoimmun Rev. 2019;18 (11):102397. doi:10.1016/j.autrev.2019.102397

25. Lubberts E. Role of T lymphocytes in the development of rheumatoid arthritis. Implications for treatment. Curr Pharm Des. 2015;21(2):142-146. doi: $10.2174 / 1381612820666140825122247$

26. Obolskiy D, Pischel I, Siriwatanametanon N, Heinrich M. Garcinia mangostana L.: a phytochemical and pharmacological review. Phytother Res. 2009;23(8):1047-1065. doi:10.1002/ptr.2730

\section{Publish your work in this journal}

Drug Design, Development and Therapy is an international, peer-reviewed open-access journal that spans the spectrum of drug design and development through to clinical applications. Clinical outcomes, patient safety, and programs for the development and effective, safe, and sustained use of medicines are a feature of the journal, which has also been accepted for indexing on PubMed Central. The manuscript management system is completely online and includes a very quick and fair peer-review system, which is all easy to use. Visit http://www.dovepress.com/testimonials.php to read real quotes from published authors.

Submit your manuscript here: https://www.dovepress.com/drug-design-development-and-therapy-journal 